\title{
Cross-sectional white matter microstructure differences in age and trait mindfulness
}

\author{
Wouter Boekel, Shulan Hsieh ${ }^{*}$ * \\ Control-Aging-Sleep-Emotion (CASE) Laboratory, Department of Psychology, College of Scoial Sciences, \\ National Cheng Kung university, Tainan, Taiwan \\ * psyhsl@mail.ncku.edu.tw
}

\section{Abstract}

The process of aging can be characterized by a decline in cognitive performance, which may be accompanied by deterioration in specific structural properties of the brain. In this study we sought to investigate to what extent mindfulness changes over the aging process, and which alterations in brain structure can be associated to aging and concomitant changes in mindfulness. We collected Mindful Attention Awareness Scale questionnaire data to assess trait mindfulness and acquired diffusion-weighted imaging data fitted to the diffusion tensor model (DTI) in a group of 97 middle-aged to elderly participants. Our results showed that trait mindfulness increased with age. In terms of white matter structure our results suggested that there was a general increase of omnidirectional diffusion, which favored radial over axial diffusivity, leading to a decrease in fractional anisotropy (FA) in older participants. We further showed that trait mindfulness mediated the FA-age effect in a localized area consisting of the internal and external capsule, as well as the corona radiata. The implication of this mediation analysis is that trait mindfulness may deter age-associated neurocognitive decline, perhaps by preventing age-associated microlesions specifically in cortico-subcortical white matter tracts. This study can be considered a pioneer of using DTI studies to investigate the relationship between age and trait mindfulness.

Accepted: October 1, 2018

Published: October 15, 2018

Copyright: @ 2018 Boekel, Hsieh. This is an open access article distributed under the terms of the Creative Commons Attribution License, which permits unrestricted use, distribution, and reproduction in any medium, provided the original author and source are credited.

Data Availability Statement: Once the manuscript is accepted, all relevant data are within the manuscript and its Supporting Information files.

Funding: This work was supported by the Ministry of Science Technology (MOST) of the Republic of China, Taiwan, for financially supporting this research (Contract No. MOST104-2410-H-006021-MY2; MOST106-2410-H-006-031-MY2).

Competing interests: The authors have declared that no competing interests exist.

\section{Introduction}

Healthy aging has become an important research topic, due to increasingly effective healthcare keeping our elders alive longer. Life expectancy has been increasing since the industrial revolution and is approaching an average of 70 years worldwide [1]. Birthrates have gone down in many countries, especially in those with low child mortality rates [1]. In the forthcoming years, wealthy countries will see larger cohorts of elderly people as compared to younger individuals. This shift in the population's age distribution increases the importance of neuropsychological research aimed at finding efficient ways of maintaining mental health in aging.

Cognitive decline is generally accepted as a "normal" part of aging. Yet, not all cognitive processes will decline with age. So far, research has shown that some cognitive processes (e.g., vocabulary, world knowledge) are less impaired while other cognitive processes such as speed of processing, working memory, and reasoning show large decrements with increasing age [2]. 
In particular, many studies have provided evidence showing that older adults have deteriorated cognitive control functions, such as less working memory capacity $[3,4,5]$, a deficit in inhibitory processing $[6,7]$, and a lack of cognitive flexibility $[8,9]$. One possible fundamental deficit that triggers these cognitive problems with increasing age is a general decrease in the control over task-relevant mental processes. That is, it becomes increasingly more difficult for older adults to apply new rules, to coordinate multiple rules, and to maintain relevant information in the context of interfering information.

More practically, age-related declines in cognitive functions have been shown to be associated with severe deficits in the elders' everyday life activities. For example, some researchers have found performance on cognitive assessments to be associated with instrumental activities of daily living (IADL), such as paying bills, shopping for groceries [10,11]. There are findings showing that updating working memory and task-switching have the strongest relationships with IADL (three domains: home management, financial management, and health and safety). There is also evidence cognitive function influences self-care capacity. For example, learning proper inhaler-use technique has been found to be associated with higher scores on the Executive Interview among older adults [12]. In addition, older adults who exhibited poor performance on tests measuring cognitive flexibility have been found to have increased difficulty in everyday problem solving.

Cognitive function and flexibility have been found to be intimately linked with mindfulness $[13,14]$. It is important to investigate whether mindfulness plays a role in the aging process. Mindfulness can be divided into two broad categories: state and trait mindfulness [15]. State mindfulness refers to how mindful an individual is in the present moment, hence reflecting a more transient, moment-to-moment experience of mindfulness [16]; whereas trait mindfulness refers to an individual's natural or innate mindfulness tendency, which has been perceived as a stable, permanent characteristic [17]. Research reporting the relationship between mindfulness and aging has focused more on the manipulation of state mindfulness rather than trait mindfulness. For example, accumulating evidence has suggested that mindfulness-based interventions (MBI; $[18,19,20])$ may provide a way to deter the detrimental effects of aging, by promoting body, cognitive, and emotional health $[21,22,13,23,24,25]$. Please note, in this study, we defined mindfulness-based intervention as a broad term, including a variety of mindfulness-related practices, such as various forms of attention-based and active-based meditations. Mindfulness-based interventions may work because they provide a more holistic attentional training, in the sense that they allow training of general attentional mechanisms, the improvement of which benefits many cognitive tasks. However, neurocognitive research on mindfulness is scarce, and the effect of mindfulness on brain structure has not been elucidated yet. Studies report neural benefits of mindfulness such as increases of overall brain fractional anisotropy (FA) [26] and altered concentrations of important brain metabolites [27] (see Fox et al., 2014 and 2016 [28,29] for reviews on the neurocognitive underpinnings of mindfulness meditation). These findings are interesting, yet there is not enough evidence to allow us to draw general conclusions.

Although research into state mindfulness has important clinical applications, trait mindfulness may provide more insight to infer how individual differences in mindfulness may interact with aging processes [17]. Based on the current literature, it still remains unclear to what extent individual differences in trait mindfulness may interact with the aging process, and whether these interactions are reflected in individual differences in brain structure and plasticity. Prior research discovering differences in the cognitive trajectories of healthy older adults [30] suggests that cognitive decline may not be a necessary consequence of aging, but may result from a range of risk factors that become more prevalent with increasing age. Understanding the mechanisms that contribute to different trajectories of cognitive decline in clinically intact 
older adults is a significant target for the prevention or reduction in progression of cognitive decline and dementia in old age.

In this study, we investigate whether trait mindfulness shows an association with age and whether brain structural differences (in terms of white matter tract) can inform us about age and mindfulness. In order to answer these questions, we recruited a group of healthy participants from middle-age to elderly and acquired their diffusion-weighted imaging (DWI) data that were further fitted to a diffusion tensor model (i.e., diffusion tensor imaging; DTI) to detect water diffusion directionality, which in turn shows the microstructural architecture of tissue. A questionnaire survey containing the Mindful Attention Awareness Scale (MAAS; [31]) was used to measure trait mindfulness.

\section{Materials and methods}

This study protocol was approved by the Human Research Ethics Committee of the National Cheng Kung University, Tainan, Taiwan, R.O.C. to protect the participants' right according to the Declaration of Helsinki and the rule of research at the University. All participants signed an informed consent form before participating in the experiments.

\section{Participants}

A total of 97 participants ( 48 females; 49 males) who were part of a larger lifespan data set collected at National Cheng Kung University were recruited for the present study. The inclusion criteria for recruitment included that participants were right-handed evaluated by Edinburgh Handedness Inventory [32], in good general health, without major neurological and psychological disorders based on the participants' self-declaration, and had normal or corrected-to-normal vision. In addition, participants with scores $<22(\mathrm{n}=4)$ on the Montreal Cognitive Assessment (MoCA) (screening for probable dementia [33]), or with scores $>13(n=7)$ on the Beck Depression Inventory II (screening for depression [34]) were excluded from the data analysis. Demographic information about the remaining 97 participants is presented in Table 1. All participants signed the informed consent form before participating in the experiments. All subjects were paid 1,500 NTD (around \$50 USD) after completion of the experiment.

\section{Questionnaires}

A set of neuropsychological questionnaires was implemented: (1) The Montreal Cognitive Assessment (MoCA) to screen participants for probable dementia [33]. (2) The Beck Depression Inventory (BDI-II) to screen depression [34]. (3) A Chinese version of the Pittsburgh Sleep Quality Index-PSQI to measure the quality and patterns of sleep [35]. (4) The 15-item

Table 1. Demographics information of pariticipants.

\begin{tabular}{l|c|c|c}
\hline & Mean & Range & SE \\
\hline Age & 57.26 & $40-77$ & 0.95 \\
\hline Education & 13.72 & $4-18$ & 0.30 \\
\hline MoCA & 26.80 & $22-30$ & 0.19 \\
\hline BDI-II & 5.12 & $0-13$ & 0.42 \\
\hline PSQI & 6.16 & $1-14$ & 0.35 \\
\hline MAAS & 61.48 & $41-90$ & 1.15 \\
\hline
\end{tabular}

Note. SE: standard error; MoCA: Montreal Cognitive Assessment; BDI-II: Beck Depression Inventory II; PSQI: Pittsburgh Sleep Quality Index; MAAS: Mindful Attention Awareness Scale.

https://doi.org/10.1371/journal.pone.0205718.t001 
Mindful Attention Awareness Scale (MAAS) to measure trait mindfulness [36]. It took between 5 and 10 minutes to complete all questionnaires.

\section{Imaging protocols}

Magnetic resonance images (MRI) were acquired using a GE MR750 3T scanner (GE Healthcare, Waukesha, WI, USA) in the Mind Research Imaging (MRI) center of National Cheng Kung University. High resolution structural images were acquired using fast-SPGR, consisting 166 axial slices (TR/TE/flip angle, $7.6 \mathrm{~ms} / 3.3 \mathrm{~ms} / 12^{\circ}$; field of view (FOV), $22.4 \times 22.4 \mathrm{~cm} 2$; matrices, $224 \times 224$; slice thickness, $1 \mathrm{~mm}$ ), and the entire process lasted for $218 \mathrm{~s}$. Diffusion weighted imaging (DWI) were obtained with a spin-echo-echo planar sequence (TR/TE $=5500$ $\mathrm{ms} /$ minimum, 50 directions with $\mathrm{b}=1000 \mathrm{~s} / \mathrm{mm} 2,100 \times 100$ matrices, slice thickness $=2.5$ $\mathrm{mm}$, voxel size $=2.5 \times 2.5 \times 2.5 \mathrm{~mm}$, number of slices $=50$, FOV $=25 \mathrm{~cm}, \mathrm{NEX}=3) .6$ non-diffusion-weighted $\left(b=0 \mathrm{~s} / \mathrm{mm}^{2}\right)$ volumes were acquired, 3 of which were acquired with reversed phase encoding so as to allow correction for susceptibility induced distortions.

\section{DTI processing}

All DWI data processing and analyses were carried out using FMRIB's Software Library (FSL, version 5.0.9; www.fmrib.ox.ac.uk/fsl; [37]). Diffusion weighted images were first converted from DICOM to NIFTI format using the MRIcron's dcm2nii tool (https://www.nitrc.org/ projects/mricron/). TOPUP [37,38] and EDDY [39] were used to clean the DWI images of artifacts caused by susceptibility induced distortions, eddy currents, and head motion. A single image without diffusion weighting ( $\mathrm{b} 0$; $\mathrm{b}$ value $=0 \mathrm{~s} / \mathrm{mm} 2$ ) was extracted from the concatenated data, and non-brain tissue was removed using FMRIB's brain extraction tool [40] to create a brain mask that was used in subsequent analyses. DTIFIT (diffusion tensor imaging fitting model; [41]) was applied to fit a tensor model at each voxel of the data to derive fractional anisotropy (FA), mean diffusivity (MD), axial diffusivity (AD), and radial diffusivity (RD) measures for further analyses.

In order to perform tract-based investigations into DTI measures we performed tract-based spatial statistics (TBSS; [42]) in FSL, which involves voxel-wise statistical analyses of the DWI data. FA images were slightly eroded and end slices were zeroed in order to remove likely outliers from the diffusion tensor fitting. The images were then nonlinearly aligned to each other and the most representative image was then identified. This target image was subsequently affine transformed to $1 \mathrm{~mm}$ MNI space. FA images were transformed to $1 \mathrm{~mm}$ MNI space using a combination of the nonlinear and affine registration. A skeletonization procedure was then performed on the group-mean FA image, the result of which was thresholded at FA $>0.2$ to identify areas most likely to belong to white matter tracts of nontrivial size.

For tract-of-interest analyses, TBSS skeletoned FA maps were overlaid with atlas masks derived from Johns Hopkins University (JHU) ICBM-DTI-81 white matter labels atlas (provided by the ICBM DTI workgroup) where 48 white matter tracts are labelled.

\section{Statistical analyses}

Linear regressions were performed in $\mathrm{R}$ (version 3.0.2; R Foundation for Statistical Computing, http://www.R-project.org) to test for (1) a linear association between participant's age and their self-reported MAAS mindfulness score, controlling for gender and MoCA, BDI, and PSQI scores as regressors of no interest; and (2) a linear association between DTI measures (i.e., FA, $\mathrm{MD}, \mathrm{RD}, \mathrm{AD}$ ) and age, controlling for gender, and MoCA, BDI, PSQI, and MAAS scores (1 regression per measure). We also performed a series of non-linear (e.g., quadratic) regression analyses which we reported the results in the supplementary figures (S1-S4 Figs). In addition, 
we performed a mediation analysis predicting age based on FA using MAAS scores as a mediator. For the Bayesian version of these tests we used the BayesFactor (http://bayesfactorpcl.rforge.r-project.org/) and BayesMed (https://CRAN.R-project.org/package=BayesMed; [43]) toolboxes. For the voxel-wise univariate regressions we used FSL's randomize function on a model mimicking our whole-brain average regressions, testing for a linear association between FA and MAAS scores, controlling for age, gender, and MoCA, BDI, and PSQI scores.

For both the behavioral and DTI results we report classical frequentist p-values, as well as Bayes factors, which provide a more conservative evaluation of the correlations. For ease of reading, we provide both $\mathrm{BF}_{10}$ (Bayes factor for the presence of a correlation) and $\mathrm{BF}_{01}$ (Bayes factor for the absence of a correlation). These are inversely related (i.e., $\mathrm{BF}_{10}=1 / \mathrm{BF}_{01}$ and $\mathrm{BF}_{01}$ $\left.=1 / \mathrm{BF}_{10}\right)$. Bayes factors may be interpreted as proportional evidence for the presence or absence of an effect. For instance $\mathrm{BF}_{10}$ of 5 may be interpreted as the data being 5 times more likely to occur under the alternative hypothesis then under the null-hypothesis. In addition, we can interpret the Bayes factor categorically based on a grouping proposed by Jeffreys [44]. Table 2 shows this evidence categorization for the $\mathrm{BF}_{01}$, edited by and taken from Wetzels and Wagenmakers ([45]; Table 1, p. 1060). For a detailed explanation of the Bayesian statistics and the Bayes factor, see [46].

\section{Results}

Minimal data set for Tables 1-4 and Figs 1-5, and the supplementary figures (S1-S4 Figs) presented in the Results section can be found in S1 File.

\section{Age and MAAS}

We performed a linear regression predicting MAAS scores based on age. Gender, MoCA, BDI, and PSQI scores were added to the GLM as regressors of no interest. A classical linear regression showed a significant association of MAAS scores $(t=3.267, p=0.00154$; Fig 1$)$ with age, suggesting that older individuals have higher mindfulness scores than younger individuals (note our sample's age range: 40-77). In addition, a Bayesian linear regression showed strong evidence of a positive association between age and MAAS $\left(\mathrm{BF}_{10}=206.9\right)$.

Table 2. Suggested categories for interpreting Bayes factors based on Jeffreys [44].

\begin{tabular}{l|l|l|l}
\hline \multicolumn{1}{l|}{ Bayes factor $\mathbf{B F}_{\mathbf{0 1}}$} & $>$ & 100 & Interpretation \\
\hline 30 & - & 100 & Extreme evidence for $\mathrm{H}_{0}$ \\
\hline 10 & - & 30 & Very Strong evidence for $\mathrm{H}_{0}$ \\
\hline 3 & - & 10 & Strong evidence for $\mathrm{H}_{0}$ \\
\hline 1 & - & 3 & Moderate evidence for $\mathrm{H}_{0}$ \\
\hline $1 / 3$ & 1 & & Anecdotal evidence for $\mathrm{H}_{0}$ \\
\hline $1 / 10$ & - & 1 & No evidence \\
\hline $1 / 30$ & - & $1 / 3$ & Anecdotal evidence for $\mathrm{H}_{1}$ \\
\hline $1 / 100$ & - & $1 / 10$ & Moderate evidence for $\mathrm{H}_{1}$ \\
\hline & - & $1 / 30$ & Strong evidence for $\mathrm{H}_{1}$ \\
\hline
\end{tabular}

https://doi.org/10.1371/journal.pone.0205718.t002 
Table 3. Mean with standard error (SE) of $\mathrm{FA} / \mathrm{MD} / \mathrm{RD} / \mathrm{AD}$ and their correlation results with age.

\begin{tabular}{|c|c|c|c|c|c|}
\hline & Mean (SE) & $\mathbf{t}$ & $p$ & $\mathrm{BF}_{10}$ & $\mathrm{BF}_{01}$ \\
\hline FA & $0.436273(0.0018885)$ & -5.497 & 0.000000357 & 21379 & 0.00004677 \\
\hline MD & $0.000761(0.0000026)$ & 4.286 & 0.0000455 & 754.7 & 0.001325 \\
\hline RD & $0.000563(0.0000029)$ & 5.033 & 0.00000246 & 9540 & 0.0001047 \\
\hline AD & $0.001134(0.0000023)$ & 1.895 & 0.0613 & 1.54641 & 0.6467 \\
\hline
\end{tabular}

\section{Age and DTI}

We performed a linear regression predicting whole brain average FA based on age while regressing out variance attributed to gender, and scores on MAAS, MoCA, BDI, and PSQI. We observed a negative correlation between FA and age $\left(t=-5.497, p=0.000000357, \mathrm{BF}_{10}=\right.$ 21379 , Table $3 \&$ Fig 2), suggesting that in older individuals the diffusion tensor is less fractionally anisotropic than in relatively younger individuals. We also extracted whole-brain skeleton-averaged $\mathrm{RD}, \mathrm{AD}$, and $\mathrm{MD}$, and regressed these against participant's age (again controlling for gender, MAAS, MoCA, BDI, and PSQI). Results can be seen in Table 3. We observed a positive relation between $\mathrm{RD}$ and age $\left(t=5.033, p=0.00000246, \mathrm{BF}_{10}=9540\right.$; Fig $3)$, and non-significant positive relation between $\mathrm{AD}$ and age $\left(t=1.895, p=0.0613, \mathrm{BF}_{10}=\right.$ 1.546). In addition, we observe a positive correlation between $\mathrm{MD}$ and age $(\mathrm{t}=4.286$, $\mathrm{p}=0.0000455, \mathrm{BF}_{10}=754.7$; Fig 4), in accordance with the increase in both $\mathrm{AD}$ and $\mathrm{RD}$.

In addition to the whole brain DTI analyses, we were also interested in exploring which white matter tracts on the skeletoned FA map might be correlated with age. We performed tract-of-interest analyses, and then partial correlated these tracts' FA with age. The results showed that 21 out of 48 tracts were significantly correlated with age (see Fig 5 for details). Most of the correlations, except the left and right superior cerebral peduncles, were negative, suggesting the majority of the tracts' FA decreased with age. We additionally performed a mass-univariate regression analysis within the whole-brain white matter skeleton in order to pinpoint spatially localized relations between age and white matter microstructure (i.e., voxelwise statistical analyses). We likewise found negative relations between age and FA in clusters of many tracts (see Fig 6 for details).

\section{DTI and MAAS}

In addition to cross-sectional age differences, we were interested in cross-sectional differences in DTI measures relating to MAAS scores. We performed a linear regression between DTI measures and MAAS, while regressing out age, gender, and scores on MoCA, BDI, and PSQI. We found no significant relation between MAAS and FA $\left(\mathrm{t}=1.095, \mathrm{p}=0.2764, \mathrm{BF}_{10}=\right.$ 0.2977). Inverting the Bayes factor here provides substantial evidence in favor of the nullhypothesis (the absence of a relation), $\mathrm{BF}_{01}=3.576$. We subsequently investigated the

Table 4. Statistical results for the correlation between FA/MD/RD/AD and the Mindful Attention Awareness Scale (MAAS).

\begin{tabular}{l|l|l|l|l} 
& $\mathbf{t}$ & $\boldsymbol{p}$ & $\mathbf{B F}_{\mathbf{1 0}}$ & $\mathbf{B F}_{\mathbf{0 1}}$ \\
\hline FA & 1.095 & 0.2764 & 0.2977 & 3.576 \\
\hline MD & 0.074 & 0.9415 & 0.3126 & 3.199 \\
\hline RD & -0.311 & 0.7563 & 0.3281 & 3.048 \\
\hline AD & 1.026 & 0.3075 & 0.2530 & 3.952 \\
\hline
\end{tabular}

https://doi.org/10.1371/journal.pone.0205718.t004 


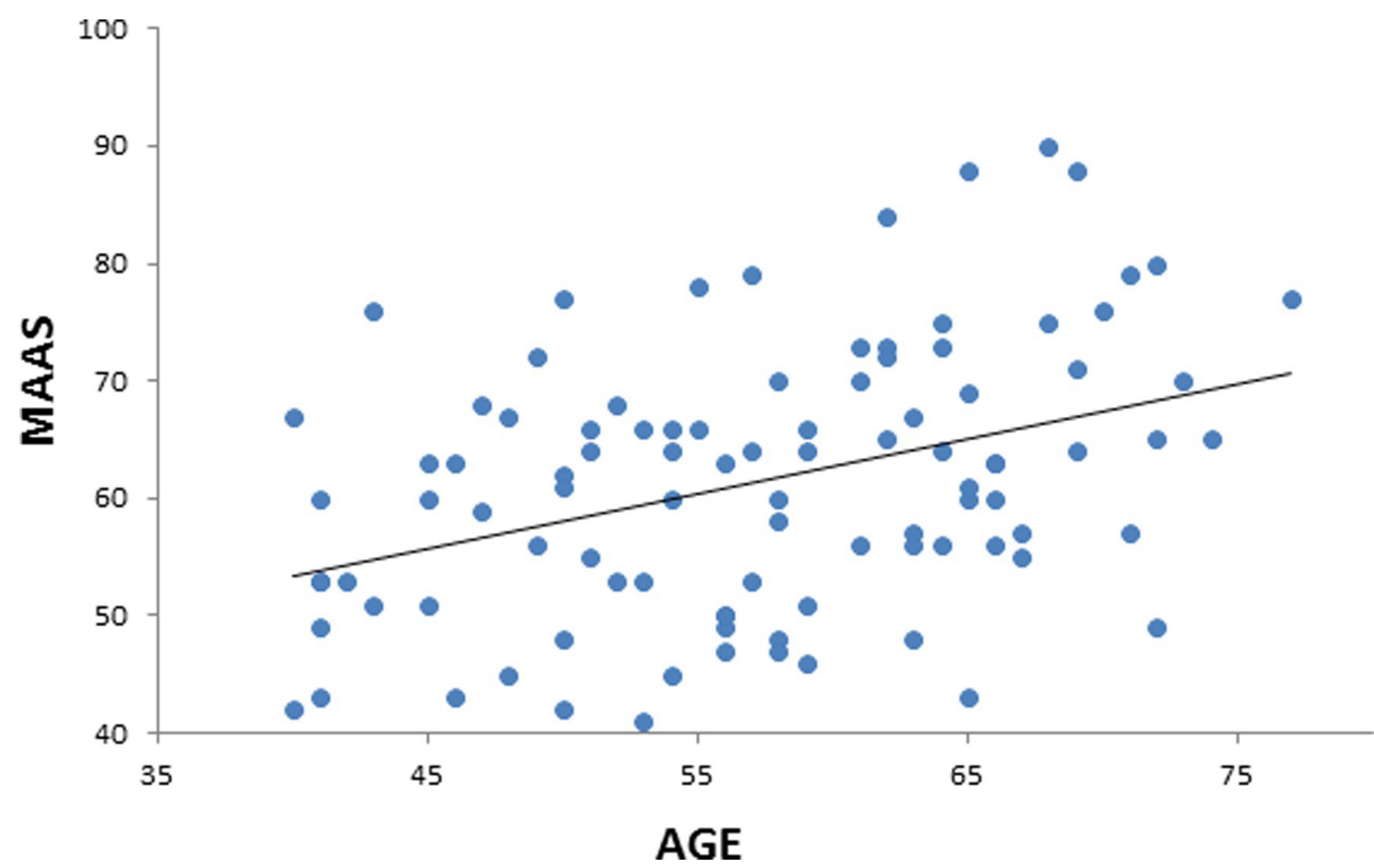

Fig 1. Positive correlation between age and mindfulness assessed by the mindful attention awareness scale (MAAS). Scatterplot with regression line.

https://doi.org/10.1371/journal.pone.0205718.g001

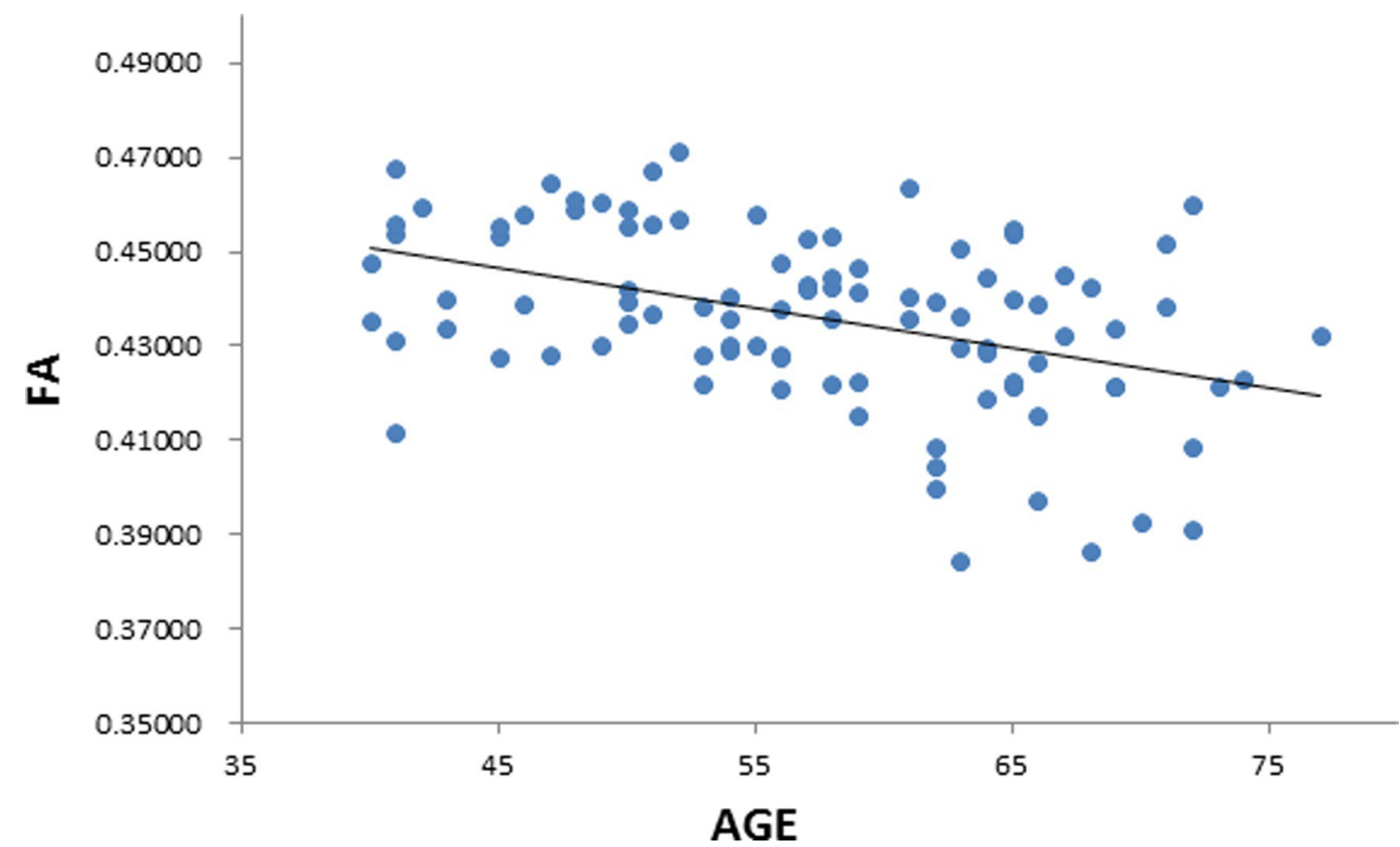

Fig 2. Negative correlation between age and fractional anisotropy (FA). Scatterplot with regression line. 


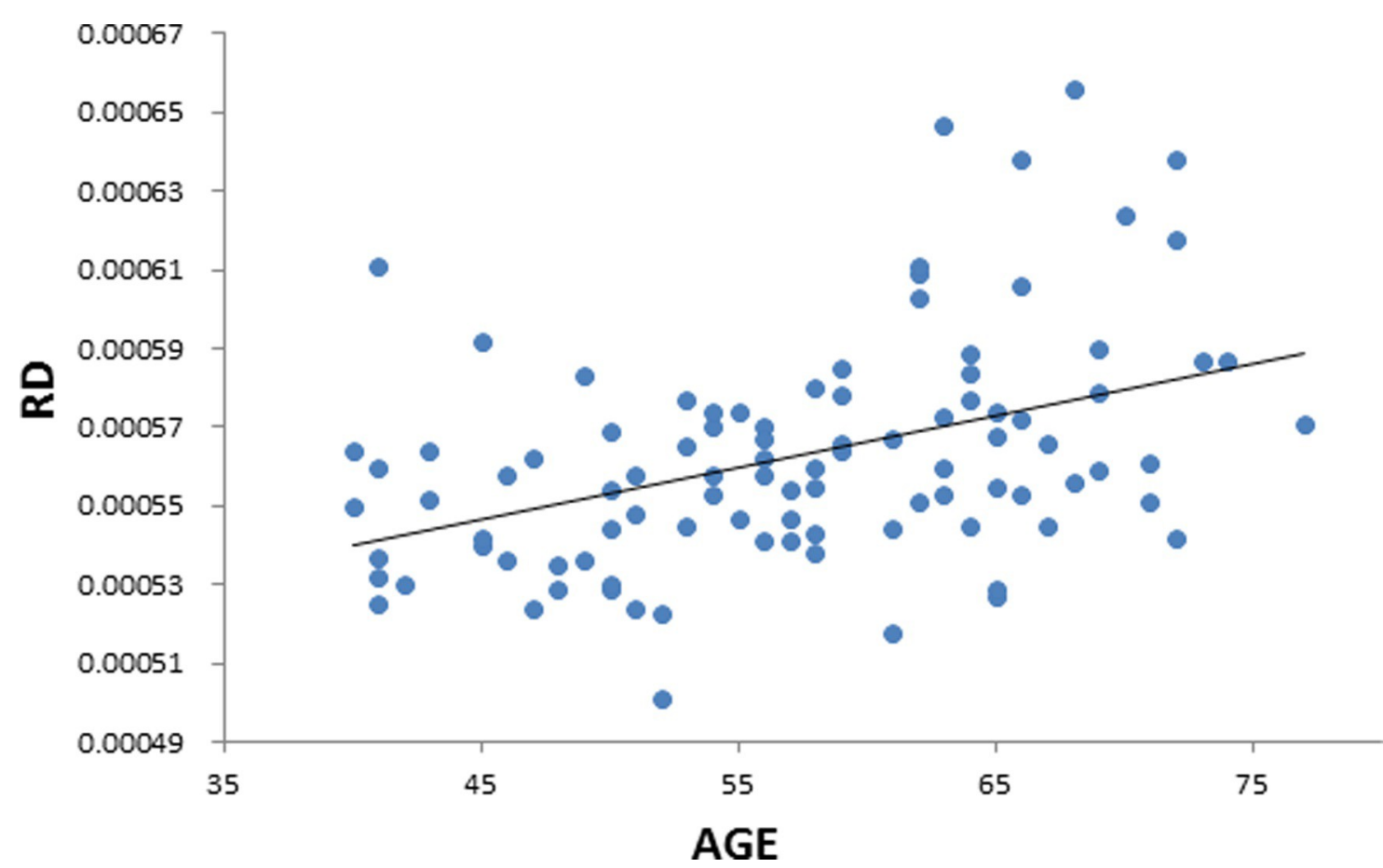

Fig 3. Positive correlation between age and radial diffusivity (RD). Scatterplot with regression line.

https://doi.org/10.1371/journal.pone.0205718.g003

remaining $\mathrm{DTI}$ measures $\mathrm{MD}, \mathrm{RD}$, and $\mathrm{AD}$, which were also found not to be associated with MAAS scores (all $\mathrm{BF}_{01}>3$, Table 4 ).

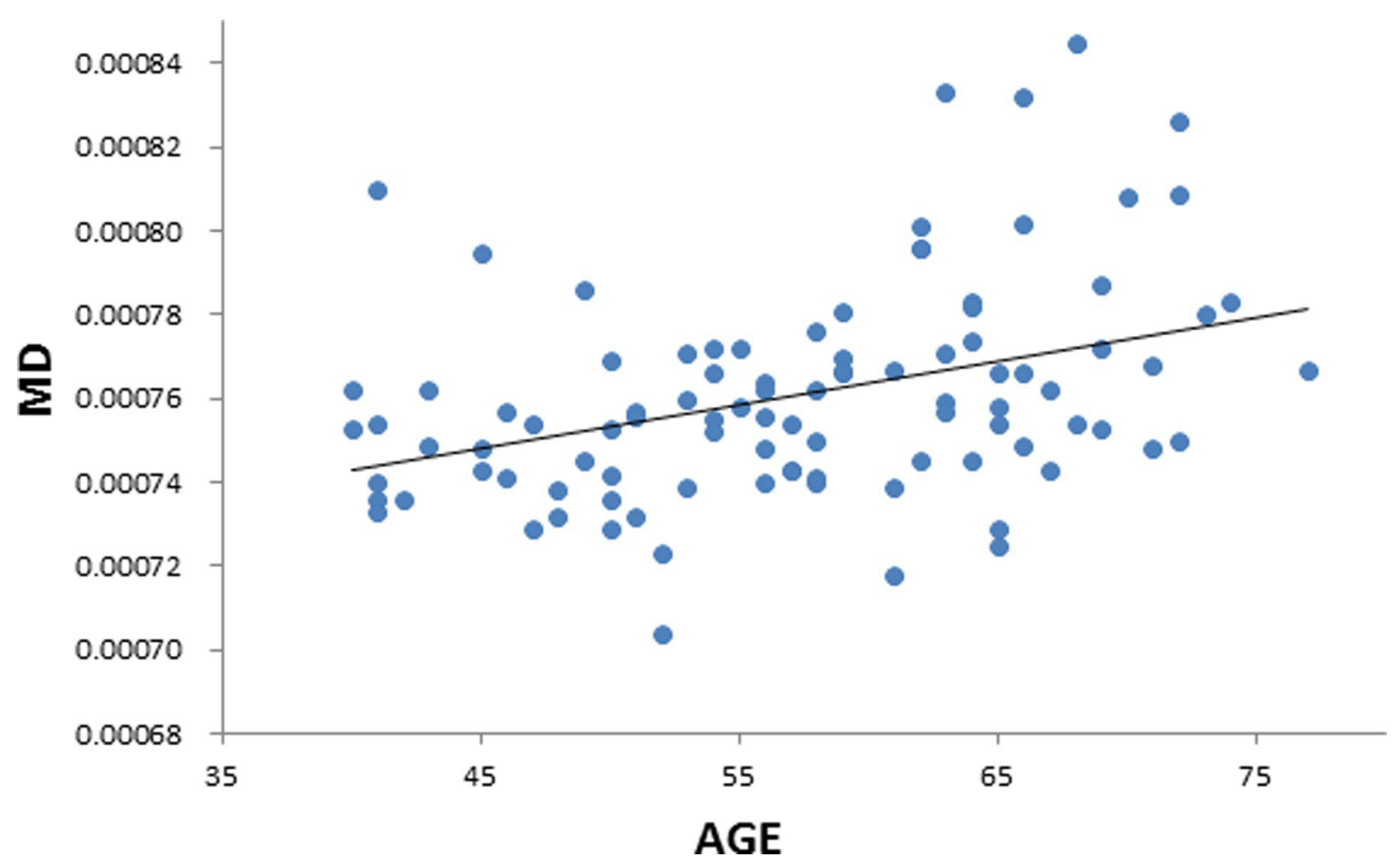

Fig 4. Positive correlation between age and mean diffusivity (MD). Scatterplot with regression line. 


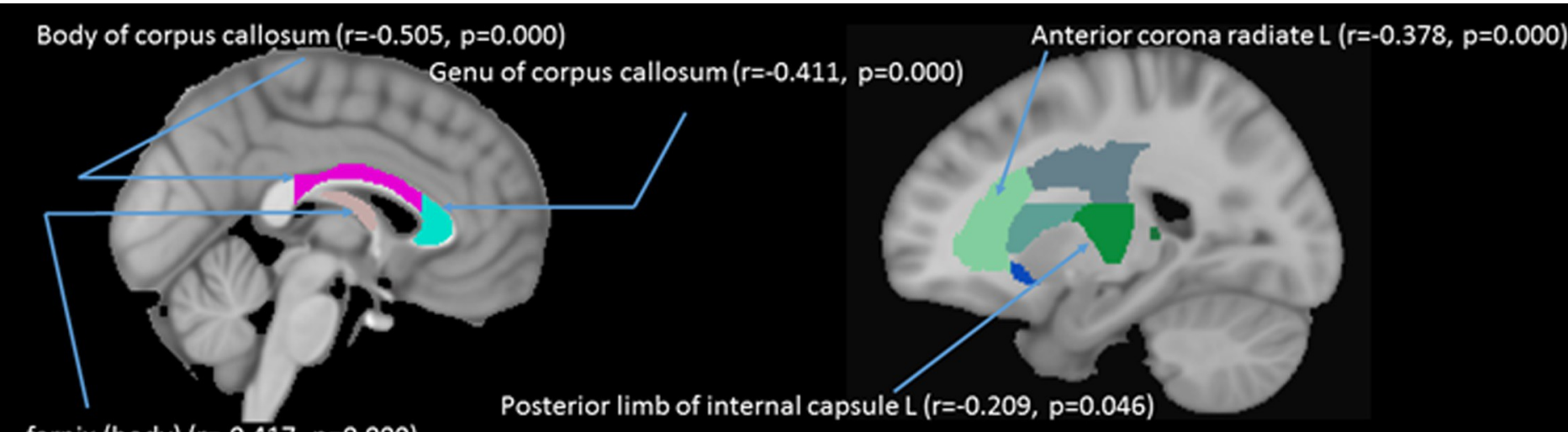

fornix (body) $(r=-0.417, p=0.000)$

Anterior limb of internal capsule $L(r=-0.218, p=0.037)$

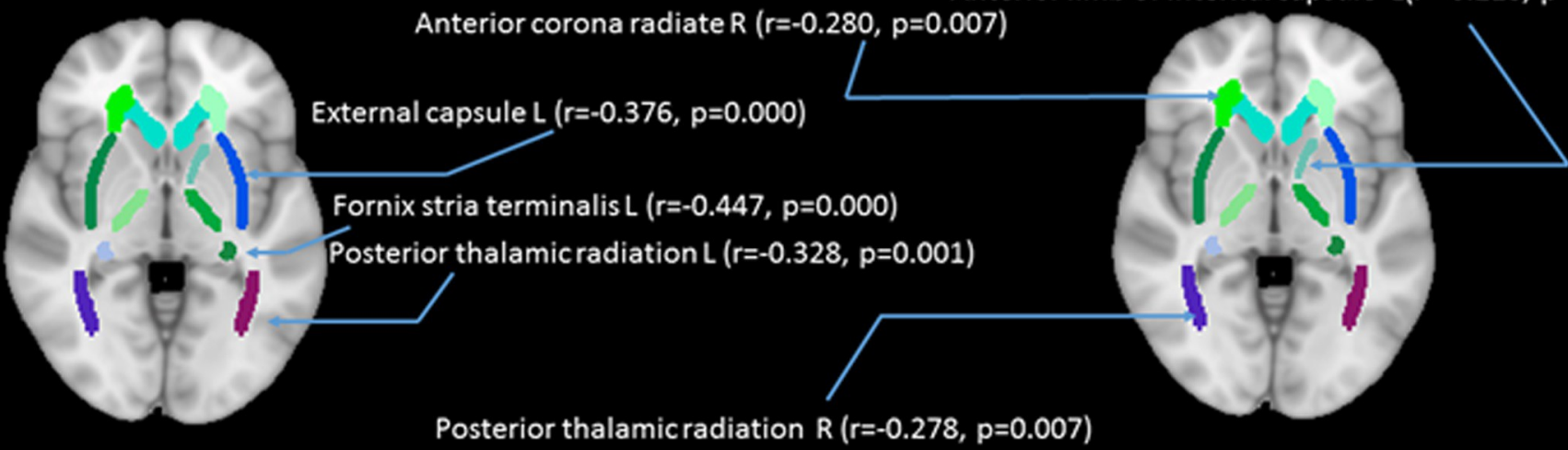

Posterior thalamic radiation $\mathrm{R}(\mathrm{r}=-0.278, \mathrm{p}=0.007)$

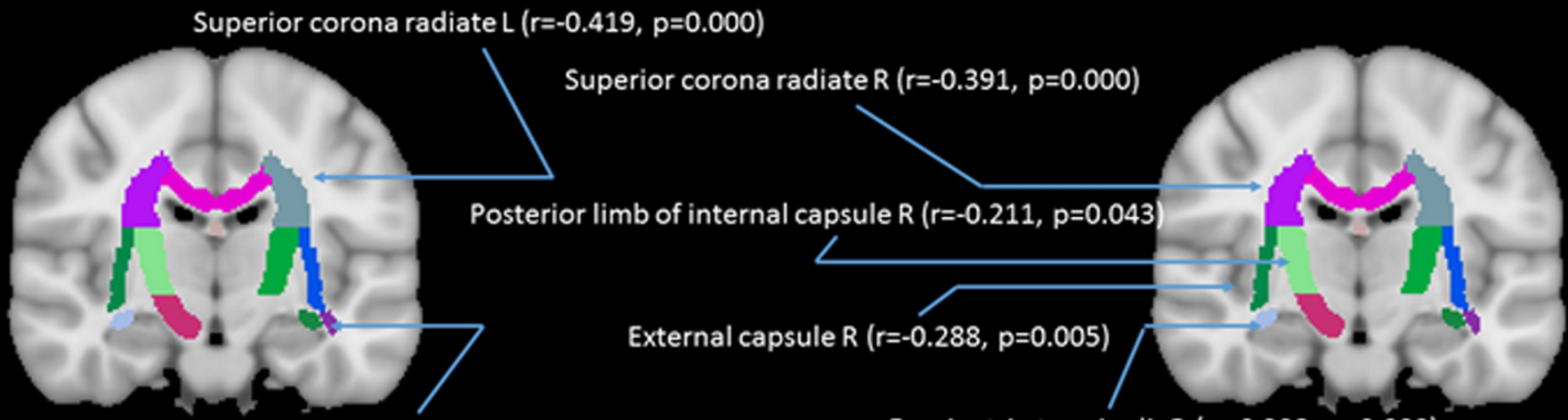

Sagittal stratum $L(r=-0.282, p=0.006)$

Fornix stria terminalis $R(r=-0.398, p=0.000)$

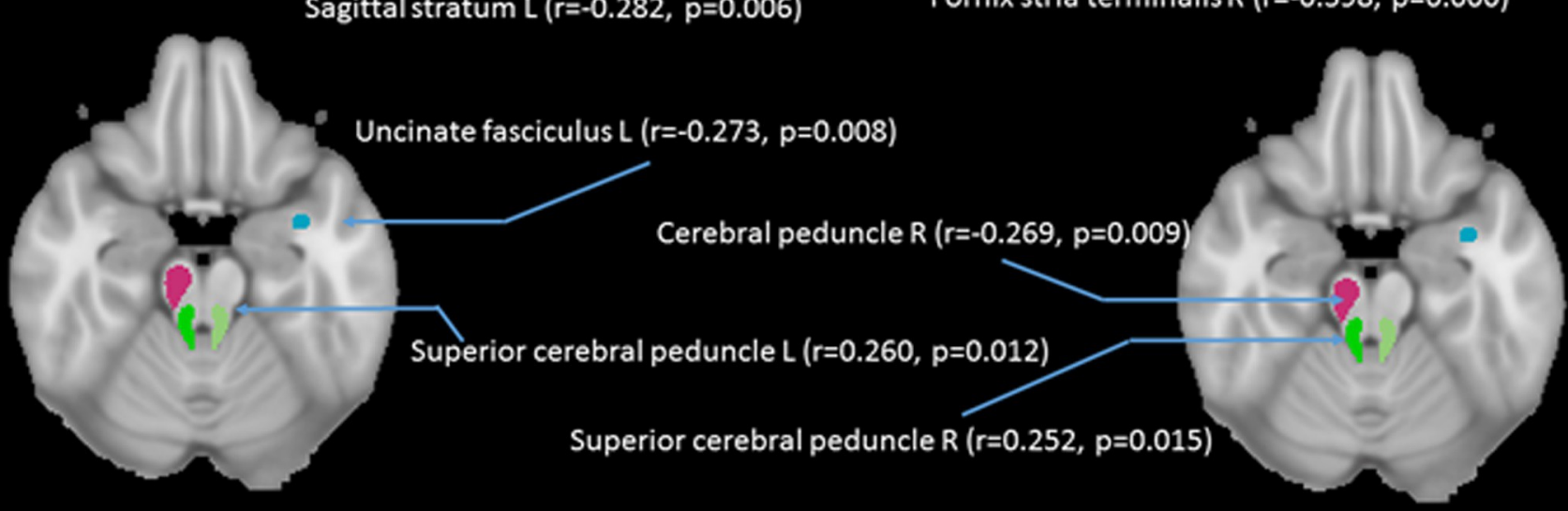

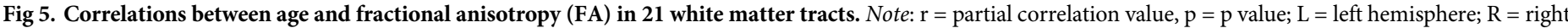
hemisphere.

https://doi.org/10.1371/journal.pone.0205718.g005 


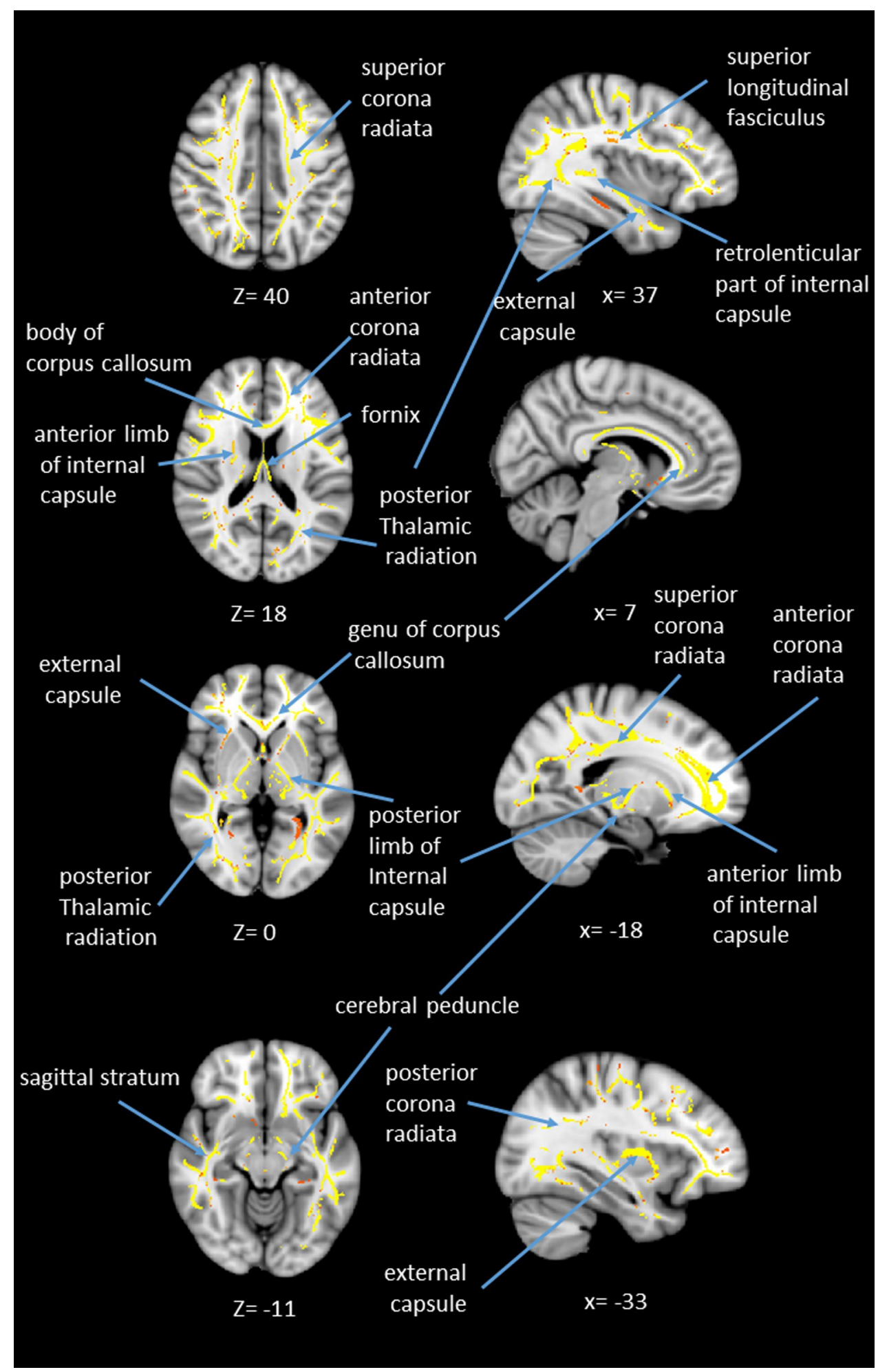

Fig 6. Statistical map showing white matter clusters (red-yellow) where fractional anisotropy (FA) was significantly correlated with age, across four axial slices (left column) and four sagittal slices (right column). Axial slices are presented in radiological orientation ( right $=$ left).

https://doi.org/10.1371/journal.pone.0205718.g006 
We performed an additional mass-univariate regression analysis within the whole-brain white matter skeleton in order to pinpoint spatially localized relations between MAAS and white matter microstructure (i.e., tensor-derived measures). We found a positive relation between FA and MAAS in the left hemisphere, specifically in the internal and external capsule, extending widely from the anterior to the posterior parts of both of these white-matter tracts, and extending dorsally into the corona radiata $(t=5.482, \mathrm{p}<0.05$ corrected for multiple comparisons; Fig 7). As mentioned previously, differences in FA can be driven by differences in $\mathrm{RD}$ as well as $\mathrm{AD}$, so we extracted the mask-average $\mathrm{AD}$ and $\mathrm{RD}$ from the voxels showing a positive relation between FA and MAAS. We found a positive relation between AD and MAAS scores $\left(\mathrm{t}=3.479, \mathrm{p}=0.000777, \mathrm{BF}_{10}=8.90\right)$, and a negative relation between $\mathrm{RD}$ and MAAS scores $\left(\mathrm{t}=-3.687, \mathrm{p}=0.000387, \mathrm{BF}_{10}=3.02\right)$. As such, it seems that in individuals with a higher self-report score on the MAAS mindfulness scale, the diffusion tensor displays a relatively decreased $\mathrm{RD}$, and a relatively increased $\mathrm{AD}$, as compared to low-mindfulness individuals, leading to an overall increase of FA in mindful individuals in the internal and external capsule extending dorsally into the corona radiata.

\section{Mediation analysis: Age, MAAS, and FA}

In light of this finding we were additionally interested in whether MAAS may serve as a mediator between age and FA within the spatially localized area which showed significant MAAS-FA associations. A mediation analysis showed that MAAS was indeed mediating the association between FA and age within this area $\left(\mathrm{BF}_{10}=45.39\right)$. We interpret this finding to suggest that as individuals age, FA in this region is preserved, especially for individuals who self-report to be more mindful.

\section{Discussion}

In this study we sought to investigate the relation between characteristics of white matter microstructure, age, and MAAS. To this end we acquired diffusion weighted imaging data in combination with a set of questionnaires (MAAS, MoCA, BDI, PSQI) from a group of elderly participants. We found that age was positively associated with MAAS suggesting that older
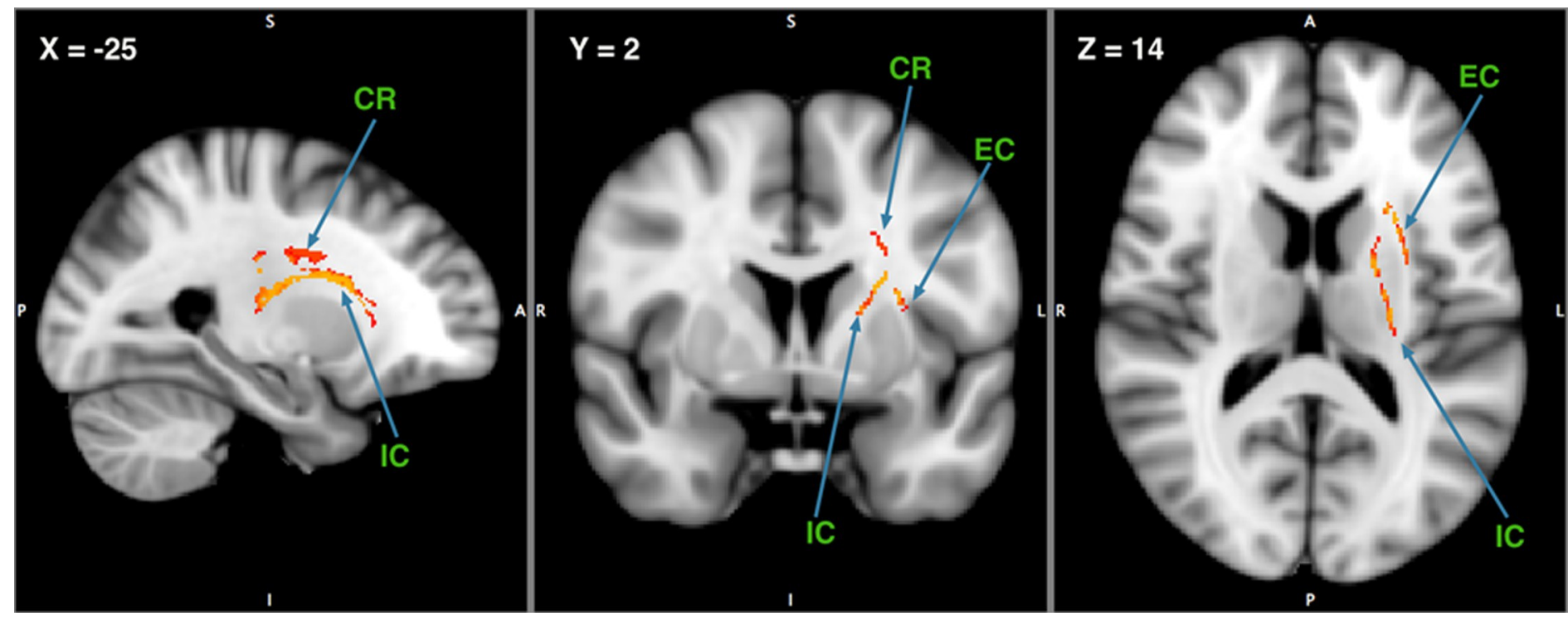

Fig 7. Statistical map showing white matter clusters (red-yellow) where MASS was positively correlated with fractional anisotropy (FA) in the internal and external capsule extending dorsally into the corona radiate (CR: Corona Radiata; IC: Internal capsule; EC: External capsule; $\mathrm{x}, \mathrm{y}, \mathrm{z}$ coordinates: -25, 2, 14).

https://doi.org/10.1371/journal.pone.0205718.g007 
individuals tend to have higher mindfulness scores. Raes et al. [47] also found a similar ageMAAS effect, as did Mahoney et al. [48], although they used a younger age range and nonlinear aging effects were observed.

In terms of age and DTI metrics our results showed a negative correlation between age and FA, suggesting that in older individuals the diffusion tensor is less fractionally anisotropic than in relatively younger individuals. Increases in FA may arise from both a relative decrease in $\mathrm{RD}$, as well as from a relative increase in $\mathrm{AD}$. In addition, $\mathrm{MD}$ provides information about the overall omnidirectional diffusion. For the purpose of understanding the nature of the tensor transformation underlying our observed FA-age effect, it is sufficient to attend to the direction of the correlation, which is positive, indicating an increase in diffusion along the principle diffusion direction (i.e., $\mathrm{AD}$ ). For the negative FA-age correlation to emerge, the RD has to sufficiently increase as well, which indeed seems to be the case. In addition, we observe a positive correlation between $\mathrm{MD}$ and age, in accordance with the increase in both $\mathrm{AD}$ and $\mathrm{RD}$. As such, we may expect that with age there is a general increase of omnidirectional diffusion, which favors radial over axial diffusivity, leading to a decrease in FA.

In addition to the whole brain DTI analyses, we also performed tract-of-interest as well as voxel-wise (randomization) analyses on FA in relation to age, the results showed that FA in many of the tracts, including major commissures (e.g., corpus callosum; fornix), radiation (e.g., corona radiate; thalamic radiation), internal/external capsule, and uncinated fasciculus, decreased with age (see Figs 5 and 6 for details). The result is consistent with previous findings which also showed a general decline in FA with age $[49,50,51,52]$.

In addition to age and DTI, we were also interested in cross-sectional differences in DTI measures relating to MAAS, and in particular whether MAAS may further serve as a mediator between age and FA. Our voxel-wise investigation into DTI metrics and MAAS found a localized positive association between FA and MAAS in the left hemisphere corona radiata and the internal and external capsule. This finding is in line with some studies using a MBI approach (a measure of state mindfulness), where FA was shown to increase in the corona radiata after intervention. For example, Tang et al [53,54] found that by training participants using a form of mindfulness meditation, integrative body-mind training (IBMT), FA increased in the corona radiata. Corona radiata is an important white matter tract connecting the anterior cingulate cortex (ACC) to other brain structures, so its communication efficiency plays a role in modulating brain activity in the ACC [55]. In line with the finding that FA increased in the corona radiata due to mindfulness mediation, Grant and colleagues [56] observed that cortical thickness in the dorsal ACC was greater for participants with experience in meditation than those without. Therefore, these findings provide convergent evidence for the important role of radiata corona in relation to state mindfulness.

In addition to the corona radiata tract and ACC, some researchers suggest that other brain regions are related to state mindfulness, including insula, temporo-parietal junction, dorsal prefrontal cortex (dPFC), ventro-medial PFC, hippocampus, amygdala, medial PFC, posterior cingulate cortex, insula, and temporo-parietal junction (see [57], Table 2). Based on this neural evidence for an association between brain structures and different facets of mindfulness meditation (e.g., intention, attention, attitude, body awareness, reappraisal, and changes in perspectives on self), Grecucci and colleagues [55] suggest that there are top-down and bottom-up neural circuits involved in the processes of mindful emotion regulation. These circuits include top-down control regions such as the PFC and the ACC and bottom-up emotional regions such as the insula (I) and the amygdala (A). Furthermore, these two neural circuits (PFC-ACC \& A-I) may interact with each other via connective neural structures such as the corona radiata. Based on the neuroimaging evidence derived from these MBI studies, the current finding that the FA in the corona radiata tract is positively correlated with MAAS appears to be 
reconciled with Grecucci et al.'s [55] postulation that the increased FA in the corona radiata tract may facilitate the connection between the two neural circuits (PFC-ACC \& A-I), resulting in FA being highly associated with MAAS. Furthermore, our additional mediation analysis showing that MAAS mediated the relationship between age and FA in the radiata corona tract, suggests that as individuals age, FA in the radiata corona is preserved, especially for those individuals with higher trait mindfulness (as reflected by MAAS scores).

It is worth noting that the current finding of a FA-MAAS association is characterized by a thinning and elongation of the diffusion tensor, this is both due to $\mathrm{AD}$ increasing with respect to MAAS, as well as $\mathrm{RD}$ decreasing. As such, it seems the white-matter in this area is more streamlined for individuals who self-report to be more mindful. The tensor model used here cannot directly inform us about the biological causes of these effects [58], although we could speculate that an overall loss of FA due to white matter lesions occurs with age [51], and that mindfulness in life may prevent some of these lesions. This speculation can be supported by the current mediation analysis showing that MAAS was indeed mediating the association between FA and age within the internal and external capsule, as well as the corona radiata. Future studies could test this hypothesis by employing mindfulness-based interventions longitudinally, since the cross-sectional nature of this study precludes a direct observation of age-related change.

Although the current findings using DTI and trait mindfulness approaches appear to be reconciled with those reported by the studies employing the MBI approach $[53,54,55,56,57]$, research into structure-function associations in trait mindfulness is still considered scarce, and the literature is not yet unified regarding the involved brain systems in state mindfulness. For example, Luders et al. [26] found larger FA in different brain structures to be associated with mindfulness training, including a fiber tract constituting the temporal component of the superior longitudinal fasciculus (tSLF) and a fiber tract linked to the hippocampus, i.e., the uncinated fasciculus (UNC). On the other hand, Tang et al. [53] and Hölzel et al. [57] additionally observed larger FA in an entire fiber tract connecting frontal and tempro-parietal regions, the SLF, and in a fiber bundle linked to the hippocampus, the cingulate cortex hippocampus $(\mathrm{CgH})$, to be associated with active mediation practices. Furthermore, in addition to the aforementioned DTI-based studies, Fox et al. [28] reviewed and used activation likelihood estimation to perform a meta-analysis on the brain structures underpinning mindfulness, in which they observed larger voxel based morphometry in intra- and inter-hemispheric communication (e.g., SLF; corpus callosum) to be associated with meditation. Given these various findings regarding which fiber tracts are related to mindfulness experience, further studies are needed to clarify the involvement of specific brain structures.

\section{Conclusion}

The current results show that with increasing age, an individual's trait mindfulness tends to increase, but in contrast, whole-brain overall FA decreases due to increasing omnidirectional diffusion. On the other hand, FA in a localized area consisting of the internal and external capsule, as well as the corona radiata, showed an increase with trait mindfulness. Interestingly, we subsequently found that trait mindfulness mediates the FA-age effect in this localized area. Therefore, we speculate that trait mindfulness may deter age-associated neurocognitive decline, perhaps by preventing age-associated microlesions specifically in cortico-subcortical white matter tracts. This study can be considered a pioneer of using DTI studies to investigate the relationship between age and trait mindfulness. 


\section{Supporting information}

S1 Fig. The supplementary figures for linear and quadratic fitting regressions for MASS and age.

(TIF)

S2 Fig. The supplementary figures for linear and quadratic fitting regressions for FA and age.

S3 Fig. The supplementary figures for linear and quadratic fitting regressions for RD and age.

S4 Fig. The supplementary figures for linear and quadratic fitting regressions for MD and age.

S1 File. Final DataSet_PLOS publish.xlsx. Minimal data set for Tables 1-4 and Figs 1-5, and the supplementary figures (S1-S4 Figs).

(XLSX)

\section{Acknowledgments}

We thank Frini Karayanidis, Birte Forstmann, Alexander Conley, and Wouter Boekel for their great help in setting up this study and Meng-Heng Yang, Hsing-Hao Lee and Yu-Chi Lin for their help in collecting data. We thank the Mind Research and Imaging Center (MRIC), supported by MOST (Ministry of Science and Technology, Taiwan), at NCKU for consultation and instrument availability. This work was financially supported by the grants (Contract No. MOST106-2410-H006-031-MY2; MOST104-2410-H-006-021-MY2) from MOST to Shulan Hsieh.

\section{Author Contributions}

Conceptualization: Shulan Hsieh.

Data curation: Shulan Hsieh.

Formal analysis: Wouter Boekel, Shulan Hsieh.

Funding acquisition: Shulan Hsieh.

Investigation: Shulan Hsieh.

Methodology: Wouter Boekel, Shulan Hsieh.

Project administration: Shulan Hsieh.

Resources: Shulan Hsieh.

Software: Shulan Hsieh.

Supervision: Shulan Hsieh.

Validation: Wouter Boekel, Shulan Hsieh.

Visualization: Wouter Boekel, Shulan Hsieh.

Writing - original draft: Wouter Boekel, Shulan Hsieh.

Writing - review \& editing: Shulan Hsieh. 


\section{References}

1. Roser M. Life Expectancy. 2017 in: OurWorldInData.org [Internet]. Available from: https:// ourworldindata.org/life-expectancy/

2. Park DC, Bischof GN. The aging mind: neuroplasticity in response to cognitive training. Dialogues Clin Neurosci. 2013; 15(1):109. PMID: 23576894

3. Grady CL, Craik FI. Changes in memory processing with age. Curr Opin Neurobiol. 2000; 10(2):224231. PMID: 10753795

4. Salthouse TA. The nature of the influence of speed on adult age differences in cognition. Dev Psychol. 1994; 30(2):240.

5. West RL. An application of prefrontal cortex function theory to cognitive aging. Psychol Bull. 1996; 120 (2):272. PMID: 8831298

6. Hasher L, Zacks RT. Working memory, comprehension, and aging: A review and a new view. Psychol Learn Motiv. 22: Elsevier; 1988. p. 193-225.

7. Kramer AF, Humphrey DG, Larish JF, Logan GD. Aging and inhibition: beyond a unitary view of inhibitory processing in attention. Psychol Aging. 1994; 9(4):491. PMID: 7893421

8. Kramer AF, Hahn S, Gopher D. Task coordination and aging: Explorations of executive control processes in the task switching paradigm. Acta Psychol (Amst). 1999; 101(2-3):339-378.

9. Mayr U. Age differences in the selection of mental sets: the role of inhibition, stimulus ambiguity, and response-set overlap. Psychol Aging. 2001; 16(1):96. PMID: 11302371

10. Cahn-Weiner DA, Boyle PA, Malloy PF. Tests of executive function predict instrumental activities of daily living in community-dwelling older individuals. Appl Neuropsychol. 2002; 9(3):187-191. https://doi. org/10.1207/S15324826AN0903_8PMID: 12584085

11. Grigsby J, Kaye K, Baxter J, Shetterly SM, Hamman RF. Executive cognitive abilities and functional status among community-dwelling older persons in the San Luis Valley Health and Aging Study. J Am Geriatr Soc. 1998; 46(5):590-596. PMID: 9588372

12. Allen SC, Jain M, Ragab S, Malik N. Acquisition and short-term retention of inhaler techniques require intact executive function in elderly subjects. Age Ageing. 2003; 32(3):299-302. PMID: 12720616

13. Moore AW, Gruber T, Derose J, Malinowski P. Regular, brief mindfulness meditation practice improves electrophysiological markers of attentional control. Front Hum Neurosci. 2012; 6:18. https://doi.org/10. 3389/fnhum.2012.00018 PMID: 22363278

14. Baer RA. Mindfulness training as a clinical intervention: A conceptual and empirical review. Clin Psychol (New York). 2003; 10(2):125-143.

15. Bilevicius E, Smith SD, Kornelsen J. Resting-State Network Functional Connectivity Patterns Associated with the Mindful Attention Awareness Scale. Brain Connect. 2018; 8(1):40-48. https://doi.org/10. 1089/brain.2017.0520 PMID: 29130326

16. Tanay G, Bernstein A. State Mindfulness Scale (SMS): development and initial validation. Psychol Assess. 2013; 25(4):1286. https://doi.org/10.1037/a0034044 PMID: 24059475

17. Brown KW, Ryan RM. The benefits of being present: mindfulness and its role in psychological wellbeing. J Pers Soc Psychol. 2003; 84(4):822. PMID: 12703651

18. Cullen M. Mindfulness-based interventions: An emerging phenomenon. Mindfulness. 2011;2(3):186193.

19. Eberth J, Sedlmeier P. The effects of mindfulness meditation: a meta-analysis. Mindfulness. 2012; 3 (3):174-189.

20. Prakash RS, De Leon AA, Patterson B, Schirda BL, Janssen AL. Mindfulness and the aging brain: a proposed paradigm shift. Front Aging Neurosci. 2014; 6:120. https://doi.org/10.3389/fnagi.2014.00120 PMID: 25009492

21. Berk $L$, van Boxtel $M$, van Os J. Can mindfulness-based interventions influence cognitive functioning in older adults? A review and considerations for future research. Aging Ment Health. 2017; 21(11):11131120. https://doi.org/10.1080/13607863.2016.1247423 PMID: 27827541

22. Malinowski $P$, Moore $A W$, Mead $B R$, Gruber $T$. Mindful aging: the effects of regular brief mindfulness practice on electrophysiological markers of cognitive and affective processing in older adults. Mindfulness. 2017; 8(1):78-94. https://doi.org/10.1007/s12671-015-0482-8 PMID: 28163795

23. Sperduti $M$, Makowski $D$, Blondé $P$, Piolino $P$. Meditation and successful aging: can meditative practices counteract age-related cognitive decline? Geriatr Psychol Neuropsychiatr Vieil. 2017; 15(2):205-213. https://doi.org/10.1684/pnv.2017.0672 PMID: 28625941 
24. Zeidan F, Johnson SK, Diamond BJ, David Z, Goolkasian P. Mindfulness meditation improves cognition: Evidence of brief mental training. Conscious Cogn. 2010; 19(2):597-605. https://doi.org/10.1016/j. concog.2010.03.014 PMID: 20363650

25. Luders $E$, Cherbuin N. Searching for the philosopher's stone: promising links between meditation and brain preservation. Ann N Y Acad Sci. 2016; 1373(1):38-44. https://doi.org/10.1111/nyas.13082 PMID: 27187107

26. Luders E, Clark K, Narr KL, Toga AW. Enhanced brain connectivity in long-term meditation practitioners. Neuroimage. 2011; 57(4):1308-1316. https://doi.org/10.1016/j.neuroimage.2011.05.075 PMID: 21664467

27. Fayed N, del Hoyo YL, Andres E, Serrano-Blanco A, Bellón J, Aguilar K, et al. Brain changes in longterm zen meditators using proton magnetic resonance spectroscopy and diffusion tensor imaging: a controlled study. PLoS ONE. 2013; 8(3):e58476. https://doi.org/10.1371/journal.pone.0058476 PMID: 23536796

28. Fox KC, Nijeboer S, Dixon ML, Floman JL, Ellamil M, Rumak SP, et al. Is meditation associated with altered brain structure? A systematic review and meta-analysis of morphometric neuroimaging in meditation practitioners. Neurosci Biobehav Rev. 2014; 43:48-73. https://doi.org/10.1016/j.neubiorev.2014. 03.016 PMID: 24705269

29. Fox KC, Dixon ML, Nijeboer S, Girn M, Floman JL, Lifshitz M, et al. Functional neuroanatomy of meditation: A review and meta-analysis of 78 functional neuroimaging investigations. Neurosci Biobehav Rev. 2016; 65:208-228. https://doi.org/10.1016/j.neubiorev.2016.03.021 PMID: 27032724

30. Yaffe K, Fiocco AJ, Lindquist K, Vittinghoff E, Simonsick EM, Newman AB, et al. Predictors of maintaining cognitive function in older adults The Health ABC Study. Neurology. 2009; 72(23):2029-2035. https://doi.org/10.1212/WNL.0b013e3181a92c36 PMID: 19506226

31. MacKillop J, Anderson EJ. Further psychometric validation of the mindful attention awareness scale (MAAS). J Psychopathol Behav Assess. 2007; 29(4):289-293.

32. Oldfield RC. The assessment and analysis of handedness: the Edinburgh inventory. Neuropsychologia. 1971; 9(1):97-113. PMID: 5146491

33. Nasreddine ZS, Phillips NA, Bédirian V, Charbonneau S, Whitehead V, Collin I, et al. The Montreal Cognitive Assessment, MoCA: a brief screening tool for mild cognitive impairment. J Am Geriatr Soc. 2005; 53(4):695-699. https://doi.org/10.1111/j.1532-5415.2005.53221.x PMID: 15817019

34. Beck AT, Steer RA, Brown GK. Beck depression inventory-II. San Antonio. 1996; 78(2):490-498.

35. Buysse DJ, Reynolds CF, Monk TH, Berman SR, Kupfer DJ. The Pittsburgh Sleep Quality Index: a new instrument for psychiatric practice and research. Psychiatry Res. 1989; 28(2):193-213. PMID: 2748771

36. Black DS, Sussman S, Johnson CA, Milam J. Psychometric assessment of the mindful attention awareness scale (MAAS) among Chinese adolescents. Assessment. 2012; 19(1):42-52. https://doi.org/10. 1177/1073191111415365 PMID: 21816857

37. Smith SM, Jenkinson M, Woolrich MW, Beckmann CF, Behrens TE, Johansen-Berg H, et al. Advances in functional and structural MR image analysis and implementation as FSL. Neuroimage. 2004; 23: S208-S219. https://doi.org/10.1016/j.neuroimage.2004.07.051 PMID: 15501092

38. Andersson JL, Skare S, Ashburner J. How to correct susceptibility distortions in spin-echo echo-planar images: application to diffusion tensor imaging. Neuroimage. 2003; 20(2):870-888. https://doi.org/10. 1016/S1053-8119(03)00336-7 PMID: 14568458

39. Andersson JL, Sotiropoulos SN. An integrated approach to correction for off-resonance effects and subject movement in diffusion MR imaging. Neuroimage. 2016; 125:1063-1078. https://doi.org/10.1016/j. neuroimage.2015.10.019 PMID: 26481672

40. Smith SM. Fast robust automated brain extraction. Hum Brain Mapp. 2002; 17(3):143-155. https://doi. org/10.1002/hbm.10062 PMID: 12391568

41. Behrens TE, Woolrich MW, Jenkinson M, Johansen-Berg H, Nunes RG, Clare S, et al. Characterization and propagation of uncertainty in diffusion-weighted MR imaging. Magn Reson Med. 2003; 50(5):10771088. https://doi.org/10.1002/mrm.10609 PMID: 14587019

42. Smith SM, Jenkinson M, Johansen-Berg H, Rueckert $D$, Nichols TE, Mackay CE, et al. Tract-based spatial statistics: voxelwise analysis of multi-subject diffusion data. Neuroimage. 2006; 31(4):14871505. https://doi.org/10.1016/j.neuroimage.2006.02.024 PMID: 16624579

43. Nuijten MB, Wetzels R, Matzke D, Dolan CV, Wagenmakers E-J. A default Bayesian hypothesis test for mediation. Behav Res Methods. 2015; 47(1):85-97. https://doi.org/10.3758/s13428-014-0470-2 PMID: 24903686

44. Jeffreys H. Theory of probability,( Oxford: Oxford University Press). 1961. 
45. Wetzels R, Wagenmakers E-J. A default Bayesian hypothesis test for correlations and partial correlations. Psychon Bull Rev. 2012; 19(6):1057-1064. https://doi.org/10.3758/s13423-012-0295-x PMID: 22798023

46. Ly A, Verhagen J, Wagenmakers E-J. An evaluation of alternative methods for testing hypotheses, from the perspective of Harold Jeffreys. J Math Psychol. 2016; 72:43-55.

47. Raes AK, Bruyneel L, Loeys $T$, Moerkerke $B$, De Raedt R. Mindful attention and awareness mediate the association between age and negative affect. J Gerontol B Psychol Sci Soc Sci. 2013; 70(2):179-188.

48. Mahoney CT, Segal DL, Coolidge FL. Anxiety sensitivity, experiential avoidance, and mindfulness among younger and older adults: Age differences in risk factors for anxiety symptoms. Int J Aging Hum Dev. 2015; 81(4):217-240. https://doi.org/10.1177/0091415015621309 PMID: 26676836

49. Salat D, Tuch D, Greve D, Van Der Kouwe A, Hevelone N, Zaleta A, et al. Age-related alterations in white matter microstructure measured by diffusion tensor imaging. Neurobiol Aging. 2005; 26(8):12151227. https://doi.org/10.1016/j.neurobiolaging.2004.09.017 PMID: 15917106

50. Sullivan EV, Rohlfing T, Pfefferbaum A. Quantitative fiber tracking of lateral and interhemispheric white matter systems in normal aging: relations to timed performance. Neurobiol Aging. 2010; 31(3):464-481. https://doi.org/10.1016/j.neurobiolaging.2008.04.007 PMID: 18495300

51. Vernooij MW, de Groot M, van der Lugt A, Ikram MA, Krestin GP, Hofman A, et al. White matter atrophy and lesion formation explain the loss of structural integrity of white matter in aging. Neuroimage. 2008; 43(3):470-477. https://doi.org/10.1016/j.neuroimage.2008.07.052 PMID: 18755279

52. Jolly TA, Cooper PS, Badwi WA, Azizah S, Phillips NA, Rennie JL, et al. Microstructural white matter changes mediate age-related cognitive decline on the Montreal Cognitive Assessment (MoCA). Psychophysiology. 2016; 53(2):258-267. https://doi.org/10.1111/psyp.12565 PMID: 26511789

53. Tang Y-Y, Lu Q, Geng X, Stein EA, Yang Y, Posner MI. Short-term meditation induces white matter changes in the anterior cingulate. Proc Natl Acad Sci U S A. 2010; 107(35):15649-15652. https://doi. org/10.1073/pnas.1011043107 PMID: 20713717

54. Tang Y-Y, Lu Q, Fan M, Yang Y, Posner MI. Mechanisms of white matter changes induced by meditation. Proc Natl Acad Sci U S A. 2012; 109(26):10570-10574. https://doi.org/10.1073/pnas.1207817109 PMID: 22689998

55. Grecucci A, Pappaianni E, Siugzdaite R, Theuninck A, Job R. Mindful emotion regulation: exploring the neurocognitive mechanisms behind mindfulness. Biomed Res Int. 2015;2015.

56. Grant JA, Courtemanche J, Duerden EG, Duncan GH, Rainville P. Cortical thickness and pain sensitivity in zen meditators. Emotion. 2010; 10(1):43. https://doi.org/10.1037/a0018334 PMID: 20141301

57. Hölzel BK, Carmody J, Vangel M, Congleton C, Yerramsetti SM, Gard T, et al. Mindfulness practice leads to increases in regional brain gray matter density. Psychiatry Res. 2011; 191(1):36-43. https:// doi.org/10.1016/j.pscychresns.2010.08.006 PMID: 21071182

58. Jones DK, Knösche TR, Turner R. White matter integrity, fiber count, and other fallacies: the do's and don'ts of diffusion MRI. Neuroimage. 2013; 73:239-254. https://doi.org/10.1016/j.neuroimage.2012.06. 081 PMID: 22846632 